\title{
DETECÇÃO DE CO e CO 2 EMITIDOS POR UM MOTOR A GASOLINA DE CICLO OTTO
}

\author{
Mateus Mesquita ${ }^{1}$, Daniel Maia Pessanha ${ }^{1}$, Marcelo da Silva Sthel ${ }^{3}$, Guilherme Rodrigues Lima ${ }^{4}$, \\ Silas das Dores Alvarenga ${ }^{2}$, Wily Câmara ${ }^{2}$ e Francisco de Assis Léo Machado ${ }^{2 *}$
}

\begin{abstract}
RESUMO
MESQUITA, M.; PESSANHA, D.M.; SHTEL, M.S.; LIMA, G.R.; ALVARENGA, S.D.; CÂMARA, W.; MACHADO, F.A.L. Detecção de $\mathrm{CO}$ e $\mathrm{CO}_{2}$ emitidos por um motor a gasolina de ciclo Otto. Perspectivas Online: Exatas \& Engenharia, v. 7, n.18, p.15-21, 2017.
\end{abstract}

As alterações climáticas tem levado diversos grupos de pesquisas a discutir soluções e alternativas que possam ao menos desacelerar o desequilíbrio ambiental. Os combustíveis fósseis, por exemplo, são um dos grandes vilões do aquecimento global. O uso desenfreado de derivados do petróleo tem contribuído demasiadamente para a elevação da temperatura terrestre. Gases como o monóxido de carbono (CO) e o dióxido de carbono $\left(\mathrm{CO}_{2}\right)$ por exemplo, provenientes da combustão que ocorre em diversos tipos de motores são considerados altamente poluentes pois impactam diretamente no efeito estufa. Neste trabalho a concentração em ppm de $\mathrm{CO}$ e $\mathrm{CO}_{2}$ emitidos por um motor a gasolina foram analisados. As medidas foram realizadas com auxílio de um analisador de gás portátil com os motores operando em alta e baixa rotação. Os resultados encontrados para $\mathrm{CO}$ e $\mathrm{CO}_{2}$ em alta rotação foram menores do que em baixa rotação, em ambos os casos, os valores estão dentro do padrão estabelecido pela legislação brasileira vigente.

Palavras-chave: Monóxido de Carbono (CO); Dióxido de Carbono $\left(\mathrm{CO}_{2}\right)$; Motor de Combustão Interna. 


\begin{abstract}
The climate change suffered by the planet has caused several researchers to discuss solutions and alternatives that could slow down the environmental impact. Fossil fuels, for example, are a major contributor to global warming. The excessive use of petroleum derivatives has contributed too much to the elevation of the terrestrial temperature. Gases such as carbon monoxide $(\mathrm{CO})$ and carbon dioxide $\left(\mathrm{CO}_{2}\right)$, for example, from combustion that occurs in several

because they directly affect the greenhouse effect. In this work the concentration in ppm of $\mathrm{CO}$ and $\mathrm{CO} 2$ emitted by a gasoline engine were analyzed. The measurements were performed using a portable gas analyzer with the motors operating at high and low rotation. The results for $\mathrm{CO}$ and $\mathrm{CO} 2$ in high rotation were lower than in low rotation, in both cases, the values are within the standard established by current Brazilian legislation.
\end{abstract} types of engines are considered highly polluting

Keywords: Carbon Monoxide (CO); Carbon Dioxide $\left(\mathrm{CO}_{2}\right)$; Internal Combustion Engine.

\footnotetext{
${ }^{1}$ Institutos Superiores de Ensino do CENSA - ISECENSA - Acadêmico do Curso de Graduação em Engenharia Mecânica - Rua Salvador Correa, 139, Centro, Campos dos Goytacazes, RJ, CEP: 28035-310, Brasil;

${ }^{2}$ Institutos Superiores de Ensino do CENSA - ISECENSA - Laboratório de Engenharia Mecânica - Rua Salvador Correa, 139, Centro, Campos dos Goytacazes, RJ, CEP: 28035-310, Brasil;

${ }^{3}$ Universidade Estadual Norte Fluminense - UENF - Laboratório de Ciências Físicas - Avenida Alberto Lamego, 2000, Parque Califórnia, Campos dos Goytacazes, RJ, CEP: 28013-602, Brasil;

${ }^{4}$ Universidade Federal do Espírito Santo - Departamento de Química e Física - Alto Universitário, s/n ${ }^{\circ}$, Guararema, Alegre, ES, CEP:29500-000, Brasil
}

(*)e-mail: franciscoleomachado@gmail.com

Data de chegada: 20/05/2017 Aceito para publicação: 28/07/2017 


\section{INTRODUÇÃO}

A poluição do ar atmosférico é um problema ambiental que tem chamado a atenção de diversos grupos de pesquisa pois seus efeitos afetam diretamente à saúde da população e aos ecossistemas. Dentre as diversas fontes emissoras de gases poluentes destacam-se os veículos automotivos de combustão interna, embora esses veículos utilizem tecnologias de redução de emissões, eles apresentam uma grande contribuição para o aumento da poluição do ar atmosférico.

No Brasil em torno de $80 \%$ da população se concentram nos centros urbanos, onde consequentemente se concentra grande parte da frota automotiva brasileira (COSTA PINTO, 2005). Os poluentes emitidos pelo tubo de escapamento dos veículos são resultados da combustão incompleta que ocorre no motor. Na queima da gasolina nos motores de ciclo OTTO, os gases da exaustão são constituídos basicamente de monóxido de carbono (CO) e hidrocarbonetos (HC), estes poluentes em contato com o sistema respiratório podem produzir efeitos nocivos à saúde da população.

Para Dutra et. al (2004), o crescimento descontrolado da frota de veículos, fruto do aumento populacional, a ineficiência dos transportes coletivos e a inadequação dos sistemas viários, vêm causando um processo contínuo de saturação das vias de tráfego dos centros urbanos. Nestes locais o aumento da emissão de gases poluentes é agravado pela manutenção inadequada dos motores e pela adulteração e deterioração dos sistemas de escapamento, atingindo muitas vezes níveis de poluição superiores aos padrões aceitáveis.

Atualmente os padrões de emissões no tubo de escapamento dos veículos automotores são regidas pela Resolução 418/2009 de 25 de novembro de 2009 do CONAMA (Conselho Nacional do Meio Ambiente) e pela Instrução Normativa $\mathrm{n}^{0}$ 6, de 8 de junho de 2010 do Ibama (Instituto Brasileiro do Meio Ambiente e dos Recursos Naturais Renováveis). Esta Instrução Normativa define o procedimento de inspeção veicular e critérios para elaboração de planos de controle de poluição veicular, bem como determina limites de emissões e procedimentos para a avaliação do estado de manutenção do veículo em uso.

Levando em consideração que os veículos a gasolina tem um impacto significante na matriz energética, haja vista que a gasolina é um derivado do petróleo, neste trabalho, foram realizadas medidas de emissões de monóxido $(\mathrm{CO})$ e dióxido de carbono $\left(\mathrm{CO}_{2}\right)$ em um motor a gasolina (ciclo Otto) de bancada que pertence ao Laboratório de Engenharia Mecânica do Instituto Superior de Ensino do Censa (ISECENSA), localizado no município de Campos dos Goytacazes-RJ, no intuito de verificar se os motores atendem as normas brasileiras vigentes, desta forma, todas as medidas foram realizadas tomando como base parâmetros definidos pela Resolução 418/2009 do CONAMA.

\section{METODOLOGIA}

\subsection{Resolução $n^{0} 418$, de 25 de Novembro de 2009}

Esta resolução dispõe sobre critérios para a elaboração de Planos de Controle de Poluição Veicular PCPV e para a implantação de Programas de Inspeção e Manutenção de Veículos em Uso - I/M pelos órgãos estaduais e municipais de meio ambiente e determina novos limites de emissão e procedimentos para a avaliação do estado de manutenção de veículos em uso.

Os valores de referência para os gases emitidos pelo escapamento dos veículos estão expressos em partes por milhão (ppm), onde se adota que 10000 ppm corresponde a $1 \%$. 
Tomando como base o Anexo I desta resolução, adotamos o limite máximo de emissão de $\mathrm{CO}_{\text {corrigido }}$ como $0,3 \%$, para veículos que operam com motor do ciclo Otto à gasolina. Este limite de emissão é para veículos fabricados a partir de 2006. Ainda com base na resolução verificamos as velocidades de rotação do motor, em marcha lenta (baixa rotação) e em alta rotação. Para marcha lenta a velocidade angular deve estar na faixa de 600 a $1200 \mathrm{rpm}$ e ser estável dentro de $\pm 100 \mathrm{rpm}$. A velocidade angular em regime acelerado (alta rotação) dever ser de 2500 rpm com tolerância de $\pm 200 \mathrm{rpm}$.

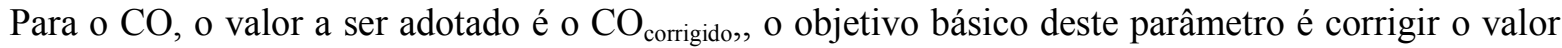
medido de CO, para obter o real emitido pelo motor e detectar vazamentos no escapamento (entrada de ar falso) através do cálculo do fator de diluição dos gases de escapamento, este valor deve ser igual ou inferior a 2,5. No caso do fator de diluição ser inferior a 1,0, este deverá ser considerado como igual a 1,0, para o cálculo do valor corrigido de CO. No caso de vazamento, o valor medido pelo CO resulta menor que o real, presente nos gases de escape.

\subsection{Fator de Diluição e $\mathrm{CO}_{\text {corrigido }}$}

A ideia do fator de diluição é que havendo diluição dos gases de escape por vazamento na tubulação de escapamento, as quantidades (em volume) de $\mathrm{CO}_{2}$ e $\mathrm{CO}$, medidas pelo analisador, resultarão tanto menor quanto maior seja a entrada de ar. Sem a presença de vazamentos e para a gasolina, a soma $\left(\mathrm{CO}+\mathrm{CO}_{2}\right)$ deve estar próxima a $15 \%$, quanto menor é o valor desta soma (com relação a 15\%), maior é a diluição. $\mathrm{Na}$ verdade, o valor de diluição é uma indicação da presença de ar falso no escape, mas não define a quantidade, em porcentagem, deste ar (Manavella, 2017). O valor de diluição real pode ser determinado pela equação 1. O CONAMA estabelece que o veículo para aprovação deve ter diluição mínima de $6 \%$.

$$
\text { Diluição real }=1-\left[\left(\% \mathrm{CO}+\% \mathrm{CO}_{2}\right) / 15\right]
$$

O valor de $\mathrm{CO}_{\text {corrigido }}$ é obtido, a partir do $\mathrm{CO}_{\text {medido, }}$ através da equação 2.

$$
\mathrm{CO}_{\text {corrigido }}=\text { F.C. } \mathrm{x} \% \mathrm{CO}_{\text {medido }} \text { com F.C. }=15 /\left[\% \mathrm{CO}_{\text {medido }}+\% \mathrm{CO}_{2}\right]
$$

O fator de correção (F.C.) serve para determinar, de forma aproximada, o CO real presente nos gases de escape, na presença de vazamento. Em um motor a gasolina, em bom funcionamento, a quantidade de $\mathrm{CO}_{2}$ gerado no escape é de aproximadamente, $15 \%$. Portanto, o fator de correção $15 /\left[\mathrm{CO}_{2}+\mathrm{CO}\right]$ é uma aproximação de quão diluídos estão os gases de escape. A ideia é que os átomos de carbono, que não são transformados em $\mathrm{CO}_{2}$, aparecem como $\mathrm{CO}$ e, portanto, a somatória deveria ser $15 \%$. Quanto maior a diluição, menor é a soma, pelo que, havendo entrada de ar falso, os valores de $\mathrm{CO}$ e $\mathrm{CO}_{2}$, medidos pelo analisador, resultam (em volume) menores que os reais emitidos pelo motor. Na ausência de diluição, os valores de $\mathrm{CO}$ medido e $\mathrm{CO}$ corrigido, resultam similares. $\mathrm{O}$ valor a ser utilizado, para certificação do

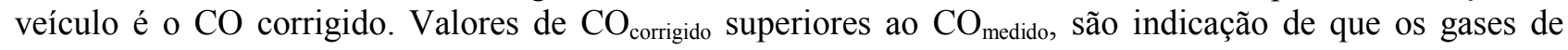
escape estão diluídos; há presença de ar falso.

\subsection{Detecção do Monóxido (CO) e Dióxido de Carbono $\left(\mathrm{CO}_{2}\right)$}

Com a utilização de um analisador de gás portátil Optima 7 (Figura 1) fabricado pela $M R U$ Air Emissions, foi identificado a quantidade em volume de monóxido $(\mathrm{CO})$ e dióxido de carbono $\left(\mathrm{CO}_{2}\right)$ emitido por um motor de bancada movido a gasolina comum. O combustível foi adquirido em um posto de gasolina

Persp. Online: exatas \& eng., Campos dos Goytacazes, 18 (07) 15 - 21 - 2017

seer.perspectivasonline.combr 
no município de Campos dos Goytacazes-RJ. O analisador é composto de sensores eletroquímicos que podem identificar até sete (7) gases distintos. A detecção dos gases no escapamento foi feita com a utilização de uma sonda, que ao ser inserido no escapamento do motor encaminha o fluxo de gases. Os gases passam por um filtro físico para a retirada de possíveis particulados e vapor d’água antes de serem detectados pelos sensores eletroquímicos em série. Após a estabilização da rotação do motor, foi estipulado um tempo de 2 minutos de detecção para cada medida, realizando três repetições em baixa e alta rotação e o valor adotado foi o valor médio.

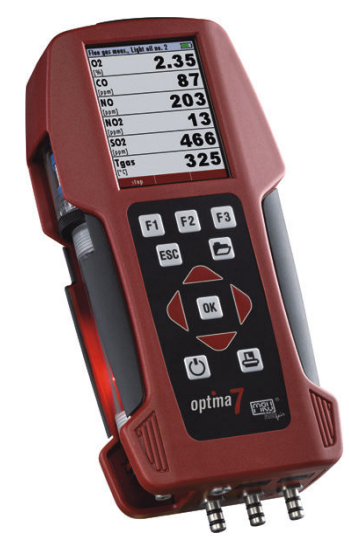

Figura 1: Analisador de Gás Portátil.

A rotação do motor foi medida com auxílio de um Tacômetro modelo MDT-2244B (Figura 2), fabricado pela Minipa. Os valor adotado para baixa rotação e alta rotação foram de $1200 \mathrm{rpm}$ e 2500 rpm, valor que está em acordo com o padrão estabelecido pela resolução do CONAMA acima citada.

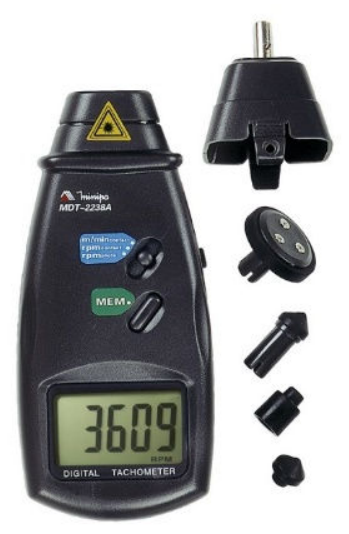

Figura 2: Tacômetro modelo MDT-2244B 


\section{RESULTADOS PARCIAIS E DISCUSSÕES}

Utilizando gasolina comum em baixa rotação (1200 rpm), os valores médios encontrados para CO e $\mathrm{CO}_{2}$, foram de $0,10 \%$ e 7,09\%, respectivamente. Substituindo esses valores na equação 2 determinamos um

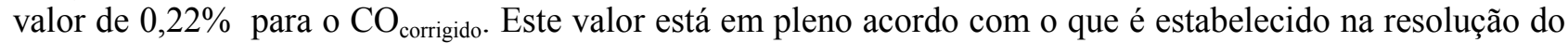
CONAMA, que recomenda que para veículos a gasolina, que utilizam motores que operam com o ciclo Otto, o valor de $\mathrm{CO}_{\text {corrigido }}$ deve ser menor ou igual a $0,3 \%$.

Em alta rotação $(2500 \mathrm{rpm})$, utilizando a mesma gasolina comum, foi observado que tanto o valor de $\mathrm{CO}$ quanto o de $\mathrm{CO}_{2}$ medidos diminuíram, $0,08 \%$ e $6.15 \%$, respectivamente. Substituindo novamente esses valores na equação 2 encontramos um valor de $0,17 \%$ para o $\mathrm{CO}_{\text {corrigido. Esses resultados também estão em }}$ acordo com a resolução do CONAMA. O que podemos observar é que o valor do $\mathrm{CO}_{\text {corrigido }}$ em alta rotação é menor do que o valor de $\mathrm{CO}_{\text {corrigido }}$ em baixa rotação. Segundo Faggi 2012, quando o motor está frio, em baixa temperatura, existe a necessidade de uma mistura rica, ou seja, a relação ar/combustível na câmara de combustão requer uma quantidade maior de combustível em relação à de ar para compensar o combustível que condensou nos dutos da admissão do motor, o fato da mistura ser mais rica em combustível, implica em uma combustão incompleta, aumentando assim a produção $\mathrm{CO}$ e $\mathrm{CO}_{2}$. Em nossos experimentos, durante a alta rotação a temperatura do motor era maior do que durante a baixa rotação, o que justifica a diminuição na produção de $\mathrm{CO}$ e $\mathrm{CO}_{2}$ e consequentemente a produção de $\mathrm{CO}_{\text {corrigido. }}$

Para Martins 2013, a mistura rica além de propiciar falta de oxigênio e combustão incompleta, pode ocasionar a formação de depósitos de carbono na câmara, nos segmentos, nas válvulas e nos eletrodos, além de aumentar o consumo de combustível, porém uma vantagem da mistura rica é que a temperatura no interior da câmara de combustível é mais baixa. A mistura quando é pobre, por apresentar uma quantidade maior de oxigênio em relação ao combustível, faz com que a temperatura da chama na vela de ignição seja mais alta, justificando a temperatura na câmara de combustão ser mais elevada na mistura pobre em relação a mistura rica.

No nosso caso, em baixa rotação, como o motor estava operando em temperaturas mais baixas que é característica de uma mistura rica, houve o aumento da emissão de $\mathrm{CO}$ e $\mathrm{CO}_{2}$.

\section{CONCLUSÕES}

$\mathrm{O}$ valor encontrado para o $\mathrm{CO}_{\text {corrigido }}$ em alta rotação, foi menor que o valor encontrado para baixa rotação. A mistura rica, que predominou durante a baixa rotação, e que apresentou menores temperaturas, favoreceu a combustão incompleta e o aumento na emissão de gases como o $\mathrm{CO}$ e o $\mathrm{CO}_{2}$. Tanto em alta rotação quanto em baixa rotação os valores de $\mathrm{CO}_{\text {medido, }}$ estão dentro do padrão estabelecido pela Resolução $\mathrm{n}^{0} 418$, de 25 de Novembro de 2009 do CONAMA.

Vale ressaltar, que durante a combustão incompleta, outros gases poluentes e nocivos à saúde humana também são emitidos pelo escapamento, tais como: Hidrocarbonetos (HC) e Dióxidos de Nitrogênio $\left(\mathrm{NO}_{\mathrm{x}}\right)$. A detecção desses gases, bem como, o estudo de emissões de gases em motores a diesel, também serão alvos de estudos deste trabalho em um futuro próximo.

Persp. Online: exatas \& eng., Campos dos Goytacazes, 18 (07) 15 - 21 - 2017

seer.perspectivasonline.com br 


\section{REFERÊNCIAS BIBLIOGRÁFICAS}

Conselho Nacional do Meio Ambiente (CONAMA), Resolução $N^{0} 418$ de 25 de Novembro de 2009.

Dutra, G. E., Valle R.M., Gomes, B.C., Fioravante, E. F., Dutra, L.G. Emissão de gases poluentes por veículos leves a gasolina na atmosfera de Belo Horizonte. Seminário de Tecnologia de Motores Combustíveis e Emissões 2004, STMCE-2004, 6 e 7 de dezembro, BH, MG.

FAGGI, R. Formação de Mistura Ar Combustível em Motores de Ignição por Faísca a Quatro Tempos. Especialização - Centro Universitário do Instituto Mauá de Tecnologia/ Programa de Engenharia Automotiva. São Caetano do Sul, 2012.

Instituto Brasileiro do Meio Ambiente e dos Recursos Naturais Renováveis (IBAMA), Instrução Normativa $\mathrm{n}^{0} 6$, de 8 de junho de 2010.

Manavella, H.J. Conceitos de Diluição e $\mathrm{CO}_{\text {corrigido }}$ Disponível em http://www.hmautotron.eng.br/artigos/Diluicao-COcorr.pdf. Acesso em: abril de 2017.

Martins, J. Motores de Combustão Interna. $4^{0}$ Edição, Porto:Publindústria, 2013. 480p.

MIGUEL, P. A. C. Estudo de caso na engenharia de produção: estruturação e recomendações para sua condução. Revista Produção, v. 17, n.1, p. 216-229, Jan. / Abr. 2007. 